\title{
Implicaciones urbanas del Tratado de Libre Comercio
}

Francisco Covarrubias Gaitán ACADEMIA MEXICANA DE ARQUITECTURA
$\mathrm{E}$ 1 Tratado de Libre Comercio tendrá una fuerte influencia en la estructura urbana, en la vivienda, en la actividad inmobiliaria, en la industria de la construcción, en la actividad propia del arquitecto y se reflejará sin duda en aspectos tan trascendentes como la cultura.

He quericlo por tanto hacer unas reflexiones sobre esta temática aun cuando, descle luego, en muchos casos tienen el carácter de conjetura y es prematuro establecer cuáles serán las condiciones y efectos del Tratado en nuestro hacer cotidiano.

Se ha manejado como el argumento central que las ventajas de la apertura comercial entre economías desiguales, en un contexto de libre comer- cio, deben elevar las condiciones de la economía menos desarrollada; $\sin \mathrm{em}$ bargo, quizá en pocos casos se ha enfrentado una situación que de partida es tan desigual.

De hecho, hoy nos encontramos ya en un esquema de apertura comercial que, desde luego, encontrará su marco definitivo en el Tratado de Libre Comercio. El aspecto fundamental es alcanzar no sólo la complementariedad, sino la competitividad.

Creo que la primera pregunta que debemos responder es cómo preparar nuestro territorio y nuestras ciudades para que el Tratado tenga efectos positivos en el bienestar del mayor número de mexicanos.

Aquí por lo tanto surgen algunos 
planteamientos que nos obligan a mirar al presente y al futuro, en algunos casos para enfrentar el rezago, en otros, para prever las implicacionesde un fenómeno complejo y con niveles de incertidumbre.

El territorio requiere de una serie de definiciones regionales, en un espacio amplio, que deben ser resultado de objetivos vinculados con el crecimiento económico $\mathrm{y}$, paralelamente, una política redistributiva que eleve los niveles de vida y atienda a las disparidades regionales.

Se presenta una disyuntiva: o podrá producirse el desarrollo equilibrado del territorio o serán necesarios cada vez mejores instrumentos para redistribuir territorialmente sus beneficios.

En gran parte, la localización de las actividades debiera responder $a$ un esquema de política territorial lo suficientemente flexible para que, en la racionalidad de la empresa, ésta pueda ubicarse con los criterios tradicionales de economías de escala, productividad y eficacia; además podemos identificar otros factores locacionales que está tomando en cuenta el capital foráneo: por una parte, la organización de la fuerza del trabajo $y$, por otra, el potencial de recursos regionales vinculados con la actividad que la propia empresa desarrolla.

En sistemas cada vez más complejos en que la complementariedad produzca economías de escala y agregación habrá otros factores que se sumen a estos criterios, entre ellos la fricción del espacio (la distancia entre los puntos de origen de los insumos y los mercados), así como las exigencias en materia de condiciones ex- ternas, particularmente de calidad del ambiente y naturaleza de la inversión.

Tradicionalmente, las líneas de in. versión extranjera han sido actividades industriales para producción y venta de productos en México, maquila para exportación, servicios turísticos y franquicias para actividades comerciales y de servicios que, desde luego, requieren de infraestructura diferente. A éstas podrá sumarse una nueva gama de inversiones en materia de servicios, que requieren nuevos espacios y proyectos vinculados con nuestra actividad gremial.

Uno de los aspectos más relevantes es cómo se pueden a provechar las ventajas de que disponemos; cómo podemos preparar nuestras ciudades para la competencia y la complementariedad, y cómo podemos prevenir problemas y riesgos que en ellas pudieran presentarse.

Si hacemos una evaluación de las condiciones necesarias en las localidades urbanas, encontraremos en principio que ciertos sistemas urbanos presentan un mayor atractivo y que es necesario precisar en cada uno de ellos la viabilidad del impulso de los centros urbanos que los conformen.

Hay ciudades que, por su localización y vocación, presentan una condición más clara para ser receptoras de inversión, en donde sus efectos multiplicadores pueden ser mayores.

Se requiere prever su capacidad de crecimiento, tomando en cuenta, tanto sus recursos naturales, particularmente la disponibilidad de agua, como aprovechar al máximo la infraestructura existente a nivel urbano e interurbano.

Así mismo, y de acuerdo con al- 
gunas experiencias en los países del Mercado Común Europeo y con otras empíricas en México, puede plantearse que habrá localidades que tengan una mayor demanda o una función específica dentro ael sistema urbano.

En nuestro caso, destaca la frontera norte que es, sin duda, una de las regiones más dinámicas del país con limitaciones, contrastes y amplias posibilidades de desarrollo.

Las ciudades medias de la frontera han experimentado cambios profundos al impulsarse su proceso de industrialización, paralelamente a la modernización del sector agrícola en sus áreas inmediatas y al crecimiento del sector de los servicios, particularmente el turismo, el comercio y los servicios transfronterizos.
En este fenómeno de urbanización, la población ha crecido con gran dinamismo dentro de un esquema de localidades concentradas, mientras que a lo largo de la línea fronteriza se presentan centros urbanos paralelos a ambos lados de la frontera.

El perfil que asi se ha producido será desde luego factor de atracción de nuevas actividades, pues estas ciudades representan una prioridad en el ordenamiento. Desde el punto de vista urbano representan implicaciones fisicas, sociales, económicas, políticas y ambientales.

Las ciudades en que se produce con mayor intensidad el proceso de industrialización a través de la maquila, habrán de cuidar, en primer lugar, la localización de esas industrias,

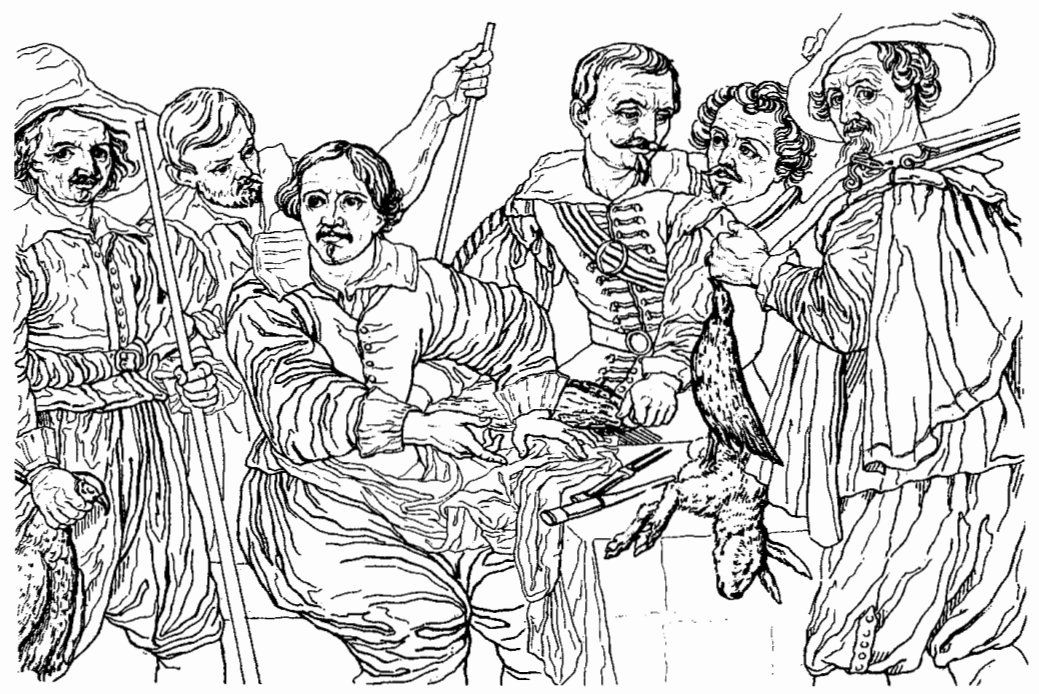

IMPLICACIONES URBANAS DEL TRATADO DE LIBRE COMERCIO 
ya que consumen energía, agua pota. ble, son origen y destino de viajes, de insumos y de personal y requieren en muchos casos de infraestructura apropiada; al mismo tiempo vierten desechos al agua, al aire y al suelo, lo que debe estar perfectamente regulado; aspectos todos que generan costos a la ciudad y que deben reflejarse en un impuesto predial apropiado.

Por lo tanto, es necesario que su localización sea congruente con los planes de desarrollo urbano en zonas o parques industriales, o bien mediante la aplicación de nuevos criterios denominados normas de comportamiento que permiten medir los efectos y requerimientos que las industrias producen y en consecuencia, lograr una ubicación apropiada.

Las ciudades de mayor dinámica industrial son también factor de atracción de la población migrante, intrarregional y de otras regiones del país. por lo que se hace necesario contar ccn planes de desarrollo actualizados que permitan dar opción de ubicación a estas industrias, al mismo tiempo que resolver alternativas de acceso al suelo, a la vivienda y a la dotación de servicios públicos; en cuanto a estos últimos, se hacen necesarios nuevos esquemas de financiamiento y operación, en particular esquemas de concesión para la prestación de servicios urbanos, previendo rezagos e incrementos.

A su vez, respecto a la tierra urbana, es necesario aprovechar las disponibilidades de tierra, en particular para uso industrial, ya que, como lo ha expresado en una serie de trabajos de investigación reciente Gustavo Garza, existen en el noreste, norte y noroeste del país, un número importante de parques industriales que habría que calificar respecto a sus atributos para recibir industria maquiladora.

Cabe destacar que, recientemente, la Secretaría de Desarrollo Urbano y Ecología anunció la adquisición de im. portantes superficies de reserva territorial: 1096 hectáreas en Tijuana; 502 en Mexicali; 109 en Nogales; 639 en Ciudad Juárez; 145 en Nuevo Laredo; 639 en Reynosa y 345 en Matamoros.

Otro aspecto que resulta de importancia fundamental, es la atención a los problemas sociales derivados de la forma de operación de la maquila, ya que en algunos casos prevalece solamente la mano de obra femenina, en edades que fluctúan de los 15 a los 25 años; es necesario así mismo atender programas de capacitación y de equipamiento social.

Los efectos en la economía urbana podrían reflejarse en unas finanzas más sanas del gobierno local; en el crecimiento de la actividad económica, secundaria y terciaria (formal e informal) y, al existir mayores niveles de circulante, es previsible que se presenten esquemas inflacionarios.

En lo político debería evitarse la creación de guetos o desarrollos privados, industriales o habitacionales fuera del control de las autoridades locales.

La industria maquiladora ha representado un gran dinamismo, particularmente en su función generadora de empleo para casi medio millón de trabajadores, al mismo tiempo que es la segunda fuente de divisas.

para definir la localización por ciu- 
dades de la industria maquiladora, hemos procesado la información a partir del Directorio Nacional de la Industria Maquiladora de Exportación, publicado por SECOFI y el Consejo Nacional de la Industria Maquiladora.

En el estado de Baja California se ubican 871 maquiladoras, correspondiendo 635 a Tijuana, 106 a Mexicali, 101 a Tecate y 36 a Ensenada.

En el estado de Sonora destaca Nogales con 80 maquiladoras, Agua Prieta con 29, Hermosillo con 26 y San Luis Río Colorado con 23.

En el estado de Chihuahua, Ciudad Juárez con 304 y la ciudad de Chihuahua con 65.

En Coahuila, Ciudad Acuña con 48; Piedras Negras con 35 y Saltillo con 9; destacando además la conurbación de La Laguna con 79 maquiladoras, que junto con Chihuahua son las localidades con más maquiladoras fuera de la frontera.

En Nuevo León existe a nivel municipal una estructura más diversificada: en Guadalupe 17 maquiladoras, en Monterrey 16, en General Escobedo 11 y en otros seis municipios un promedio de 8 maquiladoras.

En Tamaulipas las mayores concentraciones se presentan en Matamoros con 97, Nuevo Laredo con 69 y Reynosa con 67.

De hecho, la zona norte del país no es una región integrada, tanto por sus características ecológicas y morfológicas como por las distancias geográfcas; más bien se ha concebido a la franja fronteriza en términos de subregiones o microrregiones; sin embargo, se considera que los problemas que presenta son comunes y debe ser abordada en un sentido integral.

Podemos identificar dos sistemas de ciudades integrados a los extremos de la frontera; en principio el correspondiente a Tijuana, Ensenada, Mexicali, Tecate y San Luis Río Colorado, cuyas ciudades representan diversas situaciones urbanas.

En el caso de Tijuana se ha dado el mayor crecimiento urbano de la zona; persiste el problema del abasto de agua potable, igual que en Ensenada, y será necesario determinar en el corto plazo el complemento del acueducto proveniente del Río Colorado para asegurar un suministro suficiente a estas ciudades, al mismo tiempo que se concluyen importantes obras de alcantarillado y la planta binacional de tratamiento.

Tijuana tiende a conurbarse con Tecate, lo que se acelerará con la nueva carretera, lo mismo desde Rosarito hasta Ensenada; esto hace indispensable el plan regional del Corredor, ya en proceso.

Mexicali cuenta con posibilidad de agua potable y de territorio apto para su crecimiento, sin embargo su distancia hacia la costa oeste de Estados Unidos ha limitado su crecimiento; aun así, representa una alternativa importante.

Nogales por su parte aunque pudiera considerarse en general un centro aislado, representa un vínculo importante hacia el sur, que deberá fortalecerse para dar salida a los productos de Sonora, Sinaloa, Nayarit y Jalisco entre otros.

Ciudad Juárez representa una al- 
ternativa importante en la frontera, tanto para sus actividades como para su potencial de desarrollo; contará en breve con una planta de tratamiento de aguas residuales. Su situación geográfica obliga a considerarla con particular atención, ya que por un lado colinda frente al río Bravo con el estado de Texas y en las áreas inmediatas con Nuevo México, lo que hace que los inversionistas de ambos estados lo vean como un centro de interés para su inversión.

Piedras Negras y Acuña han actuado también como plazas aisladas, por lo que deberán fortalecerse sus enlaces hacia el centro, particularmente hacia Saltillo.

Por su parte, en el noreste, Nuevo Laredo, Reynosa, Río Bravo y Matamoros se identifican ya como un sistema urbano integrado con vínculos entre sí y con el área metropolitana de Monterrey, que se complementarán además, con el nuevo puente denominado Solidaridad (Colombia-Dolores) en el estado de Nuevo León.

Aparte, las comunicaciones son sin duda un factor importante en la estructuración territorial.

En el caso de Francia, se ha considerado el establecimiento de grandes corredores comerciales e industriales, que vinculen, en su caso, el sureste del país con la zona noroeste, es decir de Lille a Milán, y se ha planteado la necesidad de establecer reservas de espacio para rentabilizar y, paralelamente, un relativo dinamismo demográfico, es decir, establecer un mayor número de estrategias de desarrollo que permitan la articulación apropiada del territorio y que busque además la valori- zación de la identidad y del patrimonio cultural.

En México, nuestro sistema de comunicaciones ha sido tradicionalmente del centro a la periferia. Un objetivo fundamental debe ser propiciar la mayor articulación de las ciudades fronterizas con el resto del país, y a la vez fortalecer los ejes transversales de comunicación. Por una parte, para lograr la complementariedad de actividades y la incorporación de un mayor número de insumos nacionales; por otra, para lograr mejores flujos de comunicación que permitan que otras ciudades se beneficien del proceso y eviten una concentración extrema en las ciudades fronterizas. Los ejes transversales de comunicación deben además articular importantes zonas de producción agropecuaria.

Se hace necesario el estableçimiento de un eje transversal que integre a la frontera entre sí y anillos sucesivos que den una mayor dinámica a las ciudades dentro del territorio nacional; así, se considera que las ciudades de Monterrey, Saltillo, Torreón, Durango, Chihuahua y Hermosillo tendrán una mayor jerarquía desde el punto de vista urbano, constituyéndose en centros regionales de prestación de servicios.

Este anillo se está de hecho configurando a partir de Nuevo Laredo, Reynosa-Río Bravo; habrá un vínculo importante con Monterrey y de éste a Saltillo y la comarca Lagunera que, a su vez, se articula hacia el norte con Chihuahua y Ciudad Juárez y hacia el sur con Durango y Mazatlán; además se prevé el vínculo entre Hermosillo y Chihuahua, complementariedad que 
deberá visualizarse también, no solamente con carreteras, sino con intercomunicaciones aéreas, ferrocarril y telecomunicaciones. Este primer anillo constituirá, sin duda, un área natural de desarrollo para la industria maquiladora, apoyada además en los puertos de Topolobampo y Altamira.

Un segundo anillo lo constituye el eje transversal Manzanillo, Guadalajara, Aguascalientes, San Luis Potosí, Tampico-Ciudad Madero-Altamira.

Por otra parte, también podrá considerarse una mayor afluencia turística hacia las zonas cercanas a la frontera, a destinos de playa: San Fernando, Loreto-Nopoló, La Paz, Los Cabos, Mazatlán, Vallarta, La Pesca y Laguna Madre, así como a los lugares turísticos del Caribe mexicano. También algunas ciudades coloniales del centro-norte del país, como Zacatecas, podrán ser receptoras importantes del turismo. Esta perspectiva implica la necesidad de diversificar la economía de las ciudades de la frontera norte y acompasar el desarrollo industrial con las necesidades sociales.

A mayor distancia la actividad maquiladora se ubida en Aguascalientes con 17 maquiladoras; en La Paz, Baja California, con 14; en Guadalajara con 33 ; en el estado de Guanajuato con 26; en Puebla con 17; en Querétaro con 12 y en el área metropolitana de la ciudad de México con 26.

Se considera que estas áreas deben prever también el impacto y que

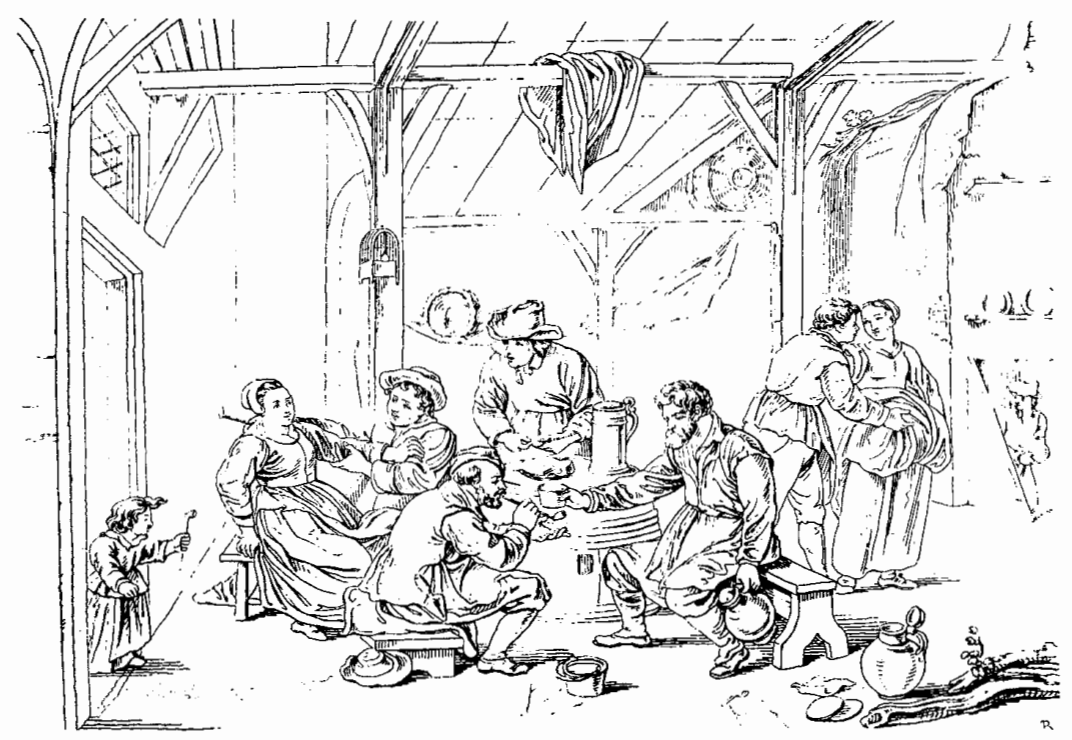


en particular las zonas metropolitanas de Guadalajara y Monterrey, deberán tener una estrategia para una ubicación periférica en las ciudades cercanas que brinden las mismas oportunidades que la propia ciudad, pero que ayuden a constituir un esquema microrregional, reduciendo su tendencia a la concentración.

En el caso de la ciudad de México es previsible que mantenga su papel de centro de decisiones de muchas de las nuevas actividades en el territorio.

En cuanto a la materia ecológica, se ha anunciado recientemente el establecimiento de un programa integral ambiental fronterizo, que viene a complementar los esfuerzos que el gobierno de la república ha venido realizando, destacándose como antecedente las acciones de saneamiento ambiental concertadas en Tijuana y Nuevo Laredo; en esta etapa se priorizan las acciones referentes a alcantarillado, plantas de tratamiento de aguas residuales y desechos sólidos (particularmente los peligrosos), contaminación atmosférica, transporte y vialidad, integración de reservas territoriales y establecimiento de un fondo de contingencias ambientales.

Es muy importante también, como se ha planteado, establecer las medidas necesarias para una adecuada regulación de estas industrias, particularmente mediante un control adecuado de descargas que evite que puedan mezclarse los grandes volúmenes de aguas negras residuales con productos químicos o metales pesados.

En materia de vivienda se presentan dos situaciones: por una parte, la demanda concentrada derivada de nuevas actividades que generarán migración; ante esta demanda es indispensable el establecimiento de reservas territoriales en cantidad suficiente para orientar el desarrollo urbano de las ciudades. Por otra parte, ya que las actuales fórmulas de atención al problema habitacional aparentemente no responden a una dinámica de crecimiento acelerado, será necesario buscar fórmulas complementarias para que, con la participación de la propia industria de la maquila, puedan edificarse un mayor número de viviendas.

Otro aspecto que debiera analizarse es el relativo a la simplificación administrativa, tanto en las instancias gubernamentales como en las bancarias, para aplicar criterios que den un tratamiento igual a promotores de ambos lados de la frontera, y que contemplen que la legislación local en materia de desarrollo urbano y fraccionamientos debe ser aplicada en cualquier tipo de autorización; ya que, en algún caso, un promotor norteamericano planteaba que la autorización de inversión extranjera era suficiente para satisfacer los requerimientos de la realización de un desarrollo.

En materia inmobiliaria, hemos visto cómo en muchas de nuestras ciudades fronterizas e incluso en otras, algunos bienes inmuebles en fraccionamientos residenciales o turísticos, se cotizan en dólares, es decir que habrá el riesgo de que las transacciones inmobiliarias se manejen en esta moneda y que probablemente guarden alguna relación los valores inmobiliarios de ambos lados de la frontera.

Asimismo en materia inmobiliaria destaca lo determinado por el artículo 
27 constitucional que señala que en una faja de $100 \mathrm{~km}$ a lo largo de las fronteras y $50 \mathrm{~km}$ en las playas, por ningún motivo podrán adquirir los extranjeros el dominio directo de tierras y aguas; consideramos que esta figura debe persistir para asegurar la soberanía de la nación sobre su territorio. aun cuando se ha venido utilizando en forma creciente la figura del fideicomiso con un plazo de 30 años y con posibilidad de ampliarlo a un segundo periodo; estos fideicomisos debieran regularse en el caso de estar dedicados a fines productivos -industriales, comerciales o turisticos- $y$ restringirse para fines habitacionales.

En relación a la vivienda, ha habido algunos puntos de vista de grupos de iniciativa privada que han analizado la competitividad entre empresas norteamericanas y mexicanas, caracterizando a las primeras como grandes empresas frente a grandes y pequeñas naciona. les; alta especialización frente a especialización media; diversos niveles de tecnología en ambos esquemas; mano de obra media en el caso norteamericano y mano de obra intensiva en el caso mexicano; manejo de materiales no convencionales en el caso americano y de materiales más durables (tradicionales) en el caso mexicano; financiamiento más barato en Estados Unidos en relación al financiamiento más costoso de México.

También en el sector privado se ha planteado la posible obtención de líneas de crédito en el extranjero que pudieran bajar los costos de financiamiento de los proyectos; sin embargo, en mi opinión, estos aspectos estarán sujetos a los acuerdos que sobre los servicios de banco se establezcan. Sin embargo, se considera que, sobre todo en relación con créditos bancarios, deberá haber una disminución en los costos financieros que repercuta en el precio de venta de los inmuebles.

Es fundamental el respeto de las costumbres regionales; en nuestro país una familia considera la vivienda como un bien duradero y la parte fundamental de su patrimonio, en la cual se arraiga. Por el contrario, en Estados Unidos la gente llega a cambiar hasta siete veces de vivienda durante su vida.

En relación a los costos de la vivienda en Estados Unidos y, sobre todo, de vivienda prefabricada de madera, ha habido experiencias de adquisición por empresas mexicanas: una vivienda de $90 \mathrm{~m}^{2}$ y prefabricada en madera ha tenido un costo de 60000000 de pesos; desde luego, implica pagar adicionalmente su cimentación y algunos complementos como paquetes de aire acondicionado, cocina integral y muebles de baño y, aun así, el costo obtenido es menor que el de una vivienda similar hecha en México.

La producción de unidades prefabricadas alcanza en Estados Unidos un volumen cercano al millón de casas al año, por lo que pudiera despertar interés en el mercado mexicano.

En cuanto a materiales de construcción, nuestro país ha tenido un crecimiento importante en su exportación, tanto en los renglones de construcción estructural y prefabricación, obra secundaria y acabados, como en productos químicos e instalaciones eléctricas e hidrosanitarias.

Actualmente, en reciente publicación que tomó como fuente el Banco 
de México, se estima que las exportaciones del país en 1990 alcanzarón 1309 millones de dólares, contra 960 millones 352000 dólares de materiales importados; sin embargo, este intercambio de materiales cubre a toda la industria de la construcción y no específicamente a la vivienda.

En síntesis, creemos que es muy importante que se encuentren fórmulas para que la balanza en esta materia resulte favorable, es decir que el volumen de las exportaciones supere al de las importaciones.

La industria de la construcción atiende dos facetas; por un lado la correspondiente a la producción de materiales que forma parte de la industria de la transformación y, por otro, la actividad constructora; sin embargo, en ambos casos el análisis de quienes participan en dichas actividacles, coincide en considerar compleja la exportación de sus servicios o productos, en función de la diversidad y requisitos reglamentarios, fianzas y garantías.

Algunas empresas y constructores han planteado esquemas de asociación y coinversión, que les permitan rcsolver estos cucllos de botella; se requerirá una nueva dinámica en la industria, la cual implica cambios y adaptaciones; han planteado también la necesidad de una reciprociclad relativa que les dé ciertas ventajas en el tiempo, así como la selectividad de las áreas en que deben abrirse.

Sumándose a las ideas del arquitecto Arturo Mier y Terán en cuanto a tecnología en la situación actual de globalización económica, las empresas constructoras nacionales enfrentarán probablemente la competencia de capital extranjero y la oferta de tecnologías, materiales y procedimientos constructivos, quizá combininada con capacidad financiera; ante estas perspectivas es necesario revisar las prácticas que prevalecen en los procesos de trabajo y capacitar a la mano de obra para su superación.

Un cambio tecnológico en los patrones de hábitos constructivos culturales es un fenómeno que implica la aceptación y apropiación de otro sistema, de sus materiales y sus diseños. Para hacer el proyecto de integrar en dichos patrones unà tecnología, es decir la forma de hacer las cosas o, a la inversa, evitar lo que resulte peligroso de esa tecnología, hay que hacer otro proyecto; a nuestro juicio existen tecnologías no funcionales para nuestro medio y como un acto consciente y racional, debemos seleccionar y desarrollar aquellas que resulten adecuadas para nuestras condiciones.

La high tech puede servirnos para resolver algunos problemas, pero la bigh tecb que nosotros necesitamos es aquella con la cual podamos enfrentar nuestros problemas de orden social, clestacanclo el de la vivienda o el de una infraestructura que nos permita hacer innovaciones tecnológicas que reduzcan costos y eliminen desechos; cle aquí que sea fundamental maximizar y ampliar la calidad de la investigación tecnológica; ésta debe desarrollarse desde la obra misma y en las instituciones de educación superior. Son factores que vienen a integrar la parte trascenclente de nuestro hacer profesional.

La arquitectura mexicana forma parte de nuestra cultura, y debe no sola- 


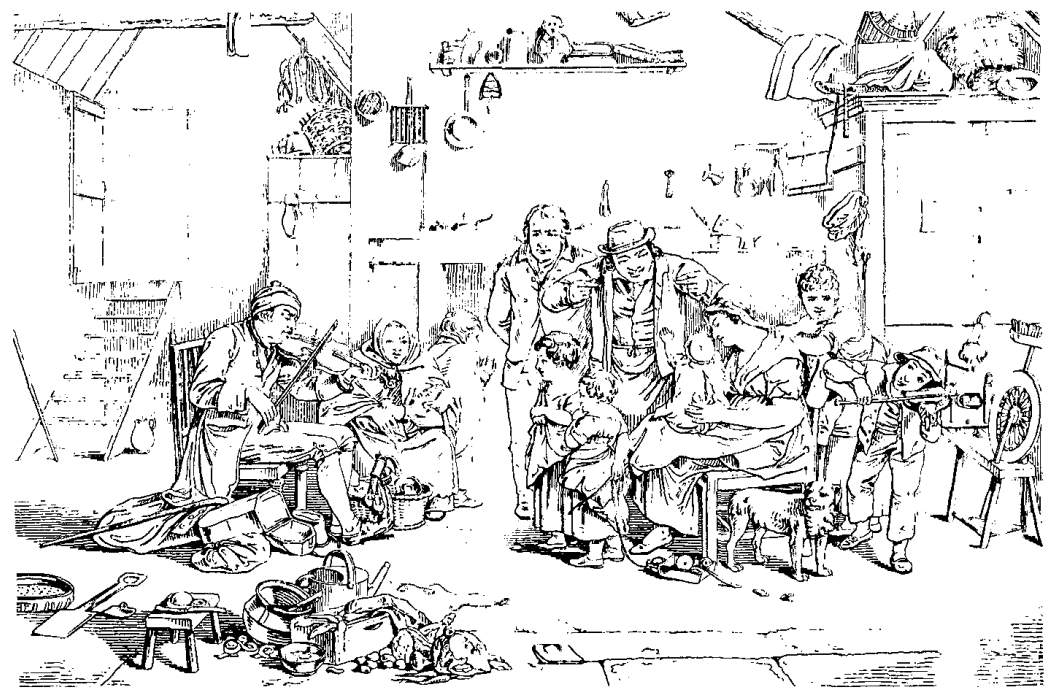

mente sustentarse en nuestro rico patrimonio arquitectónico, sino enriquecerse con cada obra que se realice en nuestras ciudades; debe ser por tanto objeto de reflexión y responsabilidad y responder a valores éticos y no sólo estéticos, dando respuesta cabal a las necesidades de nuestra sociedad.

Quisiera destacar por último la gran preocupación que ha surgido en nuestro gremio por la forma en que el trabajo profesional del arquitecto se incorporará en la apertura comercial del sector servicios; tanto el Colegio de Arquitectos de México, A. C., como la Federación de Colegios de Arquitectos, han participado en la Unidad Negociadora del Tratado en lo referente a la búsqueda de nuevos mercados y a la posible incorporación de los arqui- tectos de la zona del Tratado en nuestro contexto, buscando una situación de complementariedad y competitividad en vez de un esquema de subordinación.

Al respecto, los arquitectos Francisco Guerra Rullán a nombre del Colegio y el arquitecto Mauricio Rivero Borrel a nombre de la Federación, nos han proporcionado información sobre la posición de nuestras instituciones para este fin.

En la primera, se consideran como fundamentales los siguientes aspectos:

Que en el tratado se incorporen consideraciones sobre todas aquellas limitaciones que puedan impedir un ejercicio profesional adecuado.

Que como resultado de las negocia- 
ciones del Tratado se lleven a cabo los ajustes necesarios a la legislación para propiciar un desarrollo adecuado del ejercicio profesional, considerando las desigualdades de las economías.

Que los arquitectos de México se preparen para enfrentar este nuevo reto, por la vía de la actualización técnica y la integración profesional.

En la segunda, aunque de hecho todas son complementarias entre sí, se han planteado aspectos referentes a la educación y al ejercicio profesional del arquitecto en México y en Estados Unidos, así como a los registros, licencias, fianzas de concurso, ejecución y pago, y señalamiento de alternativas para la labor profesional del arquitecto mexicano en Estados Unidos.

A este respecto, se plantea como una alternativa la de obtener una visa de trabajo, lo cual implica un examen en cada estado norteamericano y la demostración de haber ejercido con un arquitecto reconocido y con práctica en Estados Unidos; esto, desde el principio, es un círculo vicioso. Por otra parte, en la asociación con un arquitecto residente en Estados Unidos, para ambas partes existen desventajas, por lo que se considera que no debe estar sujeto a limitaciones de un visado de trabajo.

Se plantean también, como factores fundamentales, una mayor competitividad y sistemas de organización profesional más integrados, por lo que se realizan diversas propuestas tendientes a la regulación y control de la profesión, mediante la actualización de

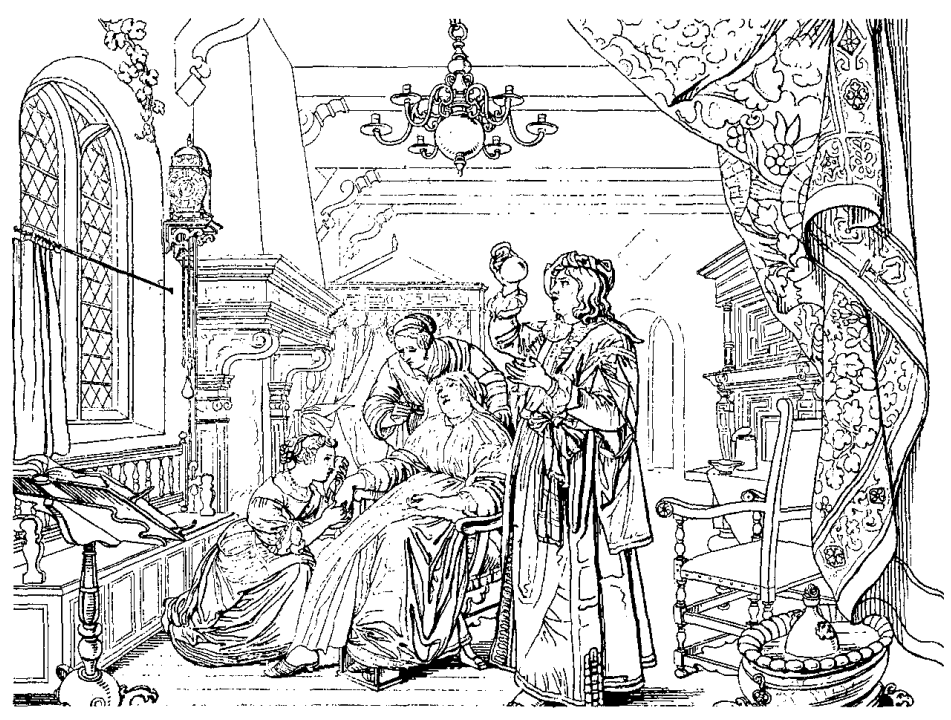


la Ley de Profesiones que debe vigilar, difundir y promover el ejercicio de la misma, la creación de comisiones técnicas de la profesión en cada estado y la conveniencia de pertenecer a un órgano colegiado.

Se plantea asimismo que el diseño y la planificación debieran incorporarse en la Ley de Obras Públicas y que los planes de desarrollo urbano y las legislaciones estatales deben estar permanentemente actualizadas. Se considera indispensable elevar la calidad de la formación y la capacitación para competir en igualdad de circunstancias.

Se plantea igualmente la liberación progresiva del mercado, es decir en un plazo de seis años; se indica que el mercado de Estados Unidos es 20 veces mayor que el de México, por lo que podría incrementarse la actividad profesional sobre una base de competencia y asociación, y no de subordinación.

Por último, se plantea un protocolo de colaboración entre la Federación de Colegios de Arquitectos, el Instituto Americano de Arquitectos y el Consejo Nacional de Comités de Registro de Arquitectura.

Como síntesis quisiera señalar:

1. La necesidad de articular el sistema de ciudades de la frontera norte con el resto del sistema urbano nacional, como un medio para consolidar la soberanía, hacer eficientes los intercambios y distribuir los beneficios del desarrollo.

2. Reordenar las ciudades de la frontera norte mediante la actualización y aplicación permanente de sus planes de desarrollo urbano, atendiendo a su dinámica con alternativas suficientes que permitan aprovechar las nuevas inversiones como factor de ordenamiento.

3. Impulsar, como lo ha propuesto la SEDUE, la creación de reservas territoriales, saneamiento fronterizo y nuevas figuras para la administración y operación de los servicios públicos.

4. Enfrentar la problemática de la vivienda con instrumentos complementarios que permitan cubrir las demandas crecientes con financiamiento y tecnología apropiada.

5. Apoyar el desarrollo de la arquitectura mexicana, como un elemento fundamental para buscar la solución de nuestros problemas de vivienda, y para que pueda atender a otros mercados en un esquema de complementariedad y competitividad, y no de subordinación. 


\section{SECUENCIG}

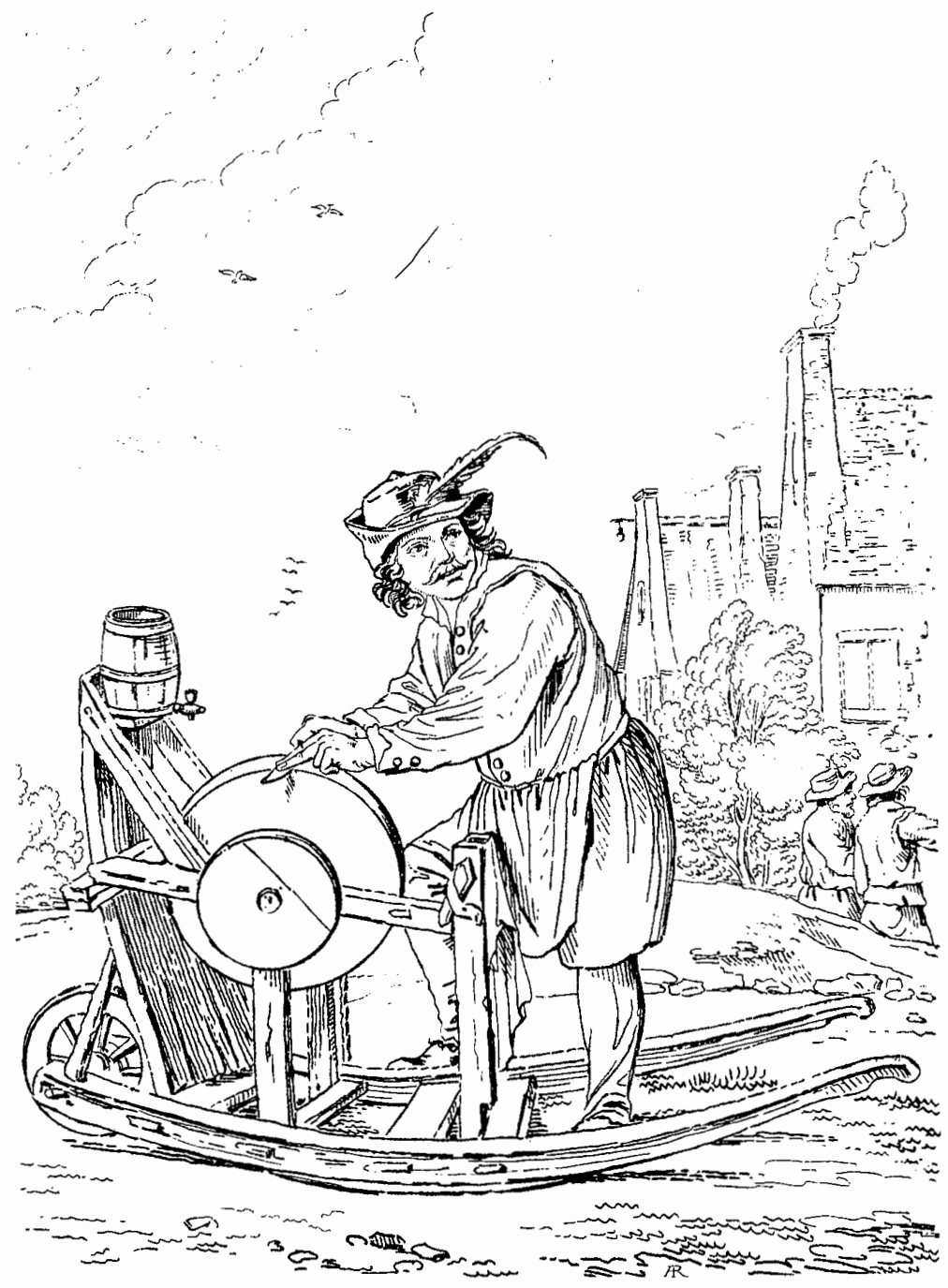

